Bull. Austral. Math. Soc.

57P10, 55м05, 57м99

VOL. 72 (2005) [331-334]

\title{
Poincaré duality pairs of dimensiond three
}

\author{
Beatrice Bleile
}

Poincaré duality complexes were introduced by Novikov and Browder [1] in the early 1960 's in connection with the surgery theory of simply connected manifolds. Later the range of applications of Poincaré duality complexes broadened. In particular, they play a crucial rôle in the surgery theory of non-simply connected manifolds developed by Wall [14].

A Poincaré duality complex of dimension $n$, or $\mathrm{PD}^{n}$-complex, is a finitely dominated $\mathrm{CW}$-complex exhibiting $n$-dimensional equivariant Poincaré duality. We may thus regard Poincaré duality complexes as homotopy generalisations of manifolds. In order to include the non-orientable case, we equip the finitely dominated $\mathrm{CW}$-complex $X$ with an element $\omega \in \mathrm{H}^{1}(X ; \mathbb{Z} / 2 \mathbb{Z})$, called the orientation character of $X$. Then the fundamental class $[X]$ of the PD ${ }^{n}$-complex $X$ is an element of the $n$-th homology of $X$ with twisted coefficients.

Every $n$-dimensional manifold is homotopy equivalent to a $C W$-complex and hence determines a $\mathrm{PD}^{n}$-complex $([10])$. But there are $\mathrm{PD}^{n}$-complexes which do not have the homotopy type of an $n$-dimensional manifold ([11] and [5]).

The classification of Poincaré duality complexes is complete in low dimensions. It is not difficult to see that every $\mathrm{PD}^{1}$-complex is homotopy equivalent to the circle. Eckmann, Müller and Linnell ([4] and [3]) showed that every group whose homology and cohomology satisfies 2-dimensional Poincaré duality, or $\mathrm{PD}^{2}$-group, is a surface group and hence every $\mathrm{PD}^{2}$-complex is homotopy equivalent to a closed surface.

As the methods of high-dimensional surgery theory are not applicable in dimension three, Poincaré duality complexes do not play the same rôle in the topology of manifolds of dimension three as they do in high dimensional topology. The main reason to studying $\mathrm{PD}^{3}$-complexes is that they represent the homotopy theory of 3-manifolds. Furthermore, they arise in situations where the geometry does not immediately provide a corresponding 3-manifold ([7]).

A finitely presentable group has $0,1,2$ or infinitely many ends. In his pioneering paper on Poincaré duality complexes [13], Wall showed that, for a $\mathrm{PD}^{3}$-complex with fundamental group $G$,

Received 22nd August, 2005

Thesis submitted to the University of Sydney, November 2004. Degree approved, February 2005. Supervisor: Dr Jonathan A. Hillman.

Copyright Clearance Centre, Inc. Serial-fee code: 0004-9727/05 $\$ A 2.00+0.00$. 
(1) if $e=0$, that is, $G$ is finite, then $X$ is finitely covered by a homotopy 3-sphere;

(2) if $e=1, \tilde{X}$ is contractible and hence $X$ is aspherical;

(3) if $e=2$, then $X$ homotopy equivalent to one of $\mathbb{R} P^{3} \sharp \mathbb{R} P^{3}, S^{1} \times \mathbb{R} P^{2}, S^{1} \times S^{2}$ or $S^{1} \tilde{x} S^{2}$;

(4) if $e$ is infinite, then $G$ decomposes as direct product if $X$ decomposes as connected sum; and if $G$ is a free product not equal to $\mathbb{Z} / 2 \mathbb{Z} * \mathbb{Z} / 2 \mathbb{Z}$, then $e$ is infinite.

Wall then asked whether a decomposition of $G$ as free product yields a decomposition of $X$ as connected sum and whether $X$ decomposes as a connected sum whenever $e$ is infinite.

Hendriks' classification theorem for $\mathrm{PD}^{3}$-complexes ([6]) together with Turaev's realisation condition ([12]) allowed Turaev to show that a $\mathrm{PD}^{3}$-complex is irreducible with respect to connected sums if and only if the fundamental group is irreducible with respect to free products, thus answering Wall's first question. Crisp [2] showed that if $e$ is infinite, then the fundamental group is either a proper free product or virtually free of finite rank. Hence every $\mathrm{PD}^{3}$-complex $X$ is finitely covered by one which is homotopy equivalent to a connected sum of aspherical $\mathrm{PD}^{3}$-complexes and copies of $S^{1} \times S^{2}$. Therefore the desired decomposition into a connected sum is available for some finite cover of $X$. But Hillman [8] recently constructed an indecomposable $\mathrm{PD}^{3}$-complex whose fundamental group has infinitely many ends and hence provided a negative answer to Wall's second question. The question whether every aspherical $\mathrm{PD}^{3}$-complex is homotopy equivalent to a 3-manifold remains open.

This thesis considers the relative case of $\mathrm{PD}^{3}$-pairs, which are the homotopy analogues of 3-manifolds with boundary. Turaev suggested generalising the results on classification, realisation and splitting to this situation, and the suggested generalisations are the content of this thesis.

We review well-known material concerning the definition and properties of the relative twisted cap products, which is not readily available in detail in the literature, as well as results on the algebraic sums of chain pairs satisfying Poincaré duality, or Poincaré chain pairs, which are needed for the discussion of connected sums of Poincaré duality pairs. One of the main results is the generalisation of a theorem by Browder [1] to the non-simply connected case.

A $\mathrm{PD}^{n}$-pair is a pair, $(X, \partial X)$, of $C W$-complexes, where $X$ is connected and finitely dominated, and $\partial X$ is a $\mathrm{PD}^{n-1}$-complex, together with an orientation character $\omega \in \mathrm{H}^{1}(X ; \mathbb{Z} / 2 \mathbb{Z})$ and a fundamental class $[X, \partial X] \in \mathrm{H}_{3}\left(X, \partial X ; \mathbb{Z}^{\omega}\right)$, such that

$$
\cap[X, \partial X]: H^{r}(X: B) \longrightarrow H_{n-r}\left(X, \partial X ; B^{\omega}\right)
$$

is an isomorphism for every $r \in \mathbb{Z}$ and every left module $B$ over the integral group ring 
of the fundamental group of $X$.

Hendriks showed that a map $f:(X, \partial X) \rightarrow(Y, \partial Y)$ of $\mathrm{PD}^{3}$-pairs with $f_{*}([X, \partial X])$ $=[Y, \partial Y]$, which induces homotopy equivalences of the boundary components and an isomorphism of $\Pi_{1}$-systems, is a homotopy equivalence of pairs. If $(X, \partial X)$ is a $\mathrm{PD}^{3}$ pair, then, up to homotopy, $X$ may be obtained by attaching a 3-cell to a homologically 2-dimensional CW-complex $X^{\prime}$. Given $\mathrm{PD}^{3}$-pairs, $(X, \partial X)$ and $(Y, \partial Y)$ and an isomorphism, $\varphi$, of their fundamental group systems with $\varphi_{*}\left(c_{X, \partial X}([X, \partial X])\right)$ $=c_{Y, \partial Y *}([Y, \partial Y])$, where $c_{X, \partial X}$ and $c_{Y, \partial Y}$ are classifying maps, we apply obstruction theory to construct a map $f^{\prime}: X^{\prime} \rightarrow Y^{\prime}$ inducing $\varphi$. Then we use homological algebra to adjust $f^{\prime}$ so that it may be extended to a map $f:(X, \partial X) \rightarrow(Y, \partial Y)$ satisfying the hypotheses of Hendriks' result. Hence the fundamental triple consisting of the $\Pi_{1}$-system

$$
\left\{\kappa_{i}: \Pi_{1}\left(\partial X_{i}, *\right) \rightarrow \Pi_{1}(X, *)\right\}_{i \in J},
$$

the orientation character $\omega_{X} \in \mathrm{H}^{1}(X ; \mathbb{Z} / 2 \mathbb{Z})$ and the image of the fundamental class $[X, \partial X] \in \mathrm{H}_{3}\left(X, \partial X ; \mathbb{Z}^{\omega}\right)$ under the classifying map

$$
c:(X, \partial X) \longrightarrow K\left(\left\{\kappa_{i}\right\}_{i \in J} ; 1\right)
$$

forms a complete set of homotopy invariants for $\mathrm{PD}^{3}$-pairs. The case $\partial X=\emptyset$ yields Hendriks' classification theorem for $\mathrm{PD}^{3}$-complexes.

The question arises which triples $\left(\left\{\kappa_{i}\right\}_{i \in J}, \omega, \mu\right)$ are realised by $\mathrm{PD}^{3}$-pairs. We introduce the projective homotopy category of modules ([9]), also called the stable category, which is needed for the formulation of the realisation condition. This condition reduces to Turaev's condition for the absolute case of $\mathrm{PD}^{3}$-complexes $X$ with $\partial X=\emptyset([12])$. Given a finitely presentable group $G$ and $\omega \in \mathrm{H}^{1}(G ; \mathbb{Z} / 2 \mathbb{Z})$, Turaev defined a homomorphism

$$
\nu: \mathrm{H}_{3}\left(G ; \mathbb{Z}^{\omega}\right) \longrightarrow[F, I]
$$

where $F$ is some $\mathbb{Z}[G]$-module, $I$ is the augmentation ideal and $[A, B]$ denotes the group of homotopy classes of $\mathbb{Z}[G]$-morphisms from the $\mathbb{Z}[G]$-module $A$ to the $\mathbb{Z}[G]$-module $B$. If

$$
\left(\left\{\kappa_{i}: G_{i} \rightarrow G\right\}_{i \in J}, \omega, \mu\right)
$$

is the fundamental triple of a $\mathrm{PD}^{3}$-pair, then $\nu(\mu)$ is a class of homotopy equivalences of $\mathbb{Z}[G]$-modules. We prove that this realisation condition is, in fact, sufficient in the case of $\Pi_{1}$-injectivity. As the assumption of $\Pi_{1}$-injectivity is indispensable for the method used, the question remains whether the realisation theorem holds without it.

Turaev used the classification theorem and the realisation condition to show that a $\mathrm{PD}^{3}$-complex decomposes as connected sum of two $\mathrm{PD}^{3}$-complexes if and only if its fundamental group decomposes as free product. There are two distinct notions of connected sum for $\mathrm{PD}^{3}$-pairs reflecting the situation of manifolds with boundary. We introduce 
the notion of interior connected sum of two $\mathrm{PD}^{3}$-pairs as well as the notion of boundary connected sum and generalise Turaev's arguments for the splitting or decomposition of $\mathrm{PD}^{3}$-complexes to both, thus obtaining splitting theorems for $\mathrm{PD}^{3}$-pairs.

\section{REFERENCES}

[1] W. Browder, Surgery on simply connected manifolds (Springer-Verlag, Berlin, Heidelberg, New York, 1972).

[2] J. Crisp, 'The decomposition of 3-dimensional Poincaré complexes', Comment. Math. Helv. 75 (2000), 232-246.

[3] B. Eckmann and P. Linnell, 'Poincaré duality groups of dimension two, II', Comment. Math. Helv. 58 (1983), 111-114.

[4] B. Eckmann and H. Müller, 'Poincaré duality groups of dimension two', Comment. Math. Helv. 55 (1980), 510-520.

[5] S. Gitler and J.D. Stasheff, 'The first exotic class of BF', Topology 4 (1965), 257-266.

[6] H. Hendriks, 'Obstruction theory in 3-dimensional topology: An extension theorem', $J$. London Math. Soc. (2) 16 (1977), 160-164.

[7] J.A. Hillman, 'On 3-dimensional Poincaré duality complexes and 2- knot groups', Math. Proc. Camb. Phil. Soc. 114 (1993), 215-218.

[8] J.A. Hillman, 'An indecomposable $P D_{3}$-complex whose fundamental group has infinitely many ends', Math. Proc. Camb. Phil. Soc 138 (2005), 55-57.

[9] P.J. Hilton, Homotopy theory and duality (Gordon and Breach, New York, London, Paris, 1965).

[10] R.C. Kirby and L.C. Siebenmann, Foundational essays on topological manifolds, smoothings and triangulations, Annals of Mathematics Studies 88 (Princeton University Press, Princeton, N.J., 1977).

[11] R.G. Swan, 'Periodic resolutions for finite groups', Ann. of Math. 72 (1960), 267-291.

[12] V.G. Turaev, 'Three dimensional Poincaré complexes: Homotopy classification and splitting', Math. Sb. 180 (1989), 809-830.

[13] C.T.C. Wall, 'Poincaré complexes: I', Ann. of Math. (2) 86 (1967), 213-245.

[14] C.T.C. Wall, Surgery on compact manifolds (Academic Press, London, New York, 1970).

School of Mathematics and Statistics

University of Sydney NSW 2006

Australia 